\title{
MIXED NORM ESTIMATES FOR A RESTRICTED X-RAY TRANSFORM
}

\author{
MICHAEL CHRIST AND M. BURAK ERDOĞAN
}

\section{In memory of Thomas H. Wolff}

In this paper we establish optimal mixed norm inequalities, except for endpoints, for a certain restricted X-ray transform in arbitrary dimensions. In doing so, we demonstrate that a method [2] applied heretofore only to simpler nonmixed norm estimates can also be adapted to the mixed norm case.

For each $\theta$ in a fixed compact subinterval $I_{0}$ of $\mathbb{R}^{1}$, define $\nu_{\theta}=\left(1, \theta, \theta^{2}, \ldots, \theta^{d-1}\right)$ and $V_{\theta}=\left\{y \in \mathbb{R}^{d}: y \perp \nu_{\theta}\right\}$. The hyperplane $V_{\theta}$ is equipped with Lebesgue measure. Define $G=\left\{(\theta, y) \in I_{0} \times \mathbb{R}^{d}: y \in V_{\theta}\right\}$. Let $f$ be defined on $\mathbb{R}^{d}$. For each $(\theta, y) \in G$, define

$$
X f(\theta, y)=\int_{|s| \leq C_{0}} f\left(y+s \nu_{\theta}\right) d s
$$

where $C_{0}$ is any constant. On $G$ we define mixed norms by

$$
\|g\|_{L^{q}\left(L^{r}\right)(G)}=\left(\int_{I_{0}}\left(\int_{V_{\theta}}|g(\theta, y)|^{r} d y\right)^{q / r} d \theta\right)^{1 / q} .
$$

Here $d y$ denotes Lebesgue measure on the hyperplane $V_{\theta}$ for each $\theta$. We write simply $L^{q}(G)$ when $r=q$.

Our main result will characterize those exponents for which $X$ maps $L^{p}\left(\mathbb{R}^{d}\right)$ boundedly to $L^{q}\left(L^{r}\right)(G)$, except for endpoints. As was shown in [3], a necessary condition for $X$ to map $L^{p}\left(\mathbb{R}^{d}\right)$ boundedly to $L^{q}\left(L^{r}\right)(G)$ is that $\left(p^{-1}, q^{-1}, r^{-1}\right)$ satisfy all three of the following inequalities:

$$
\begin{aligned}
d p^{-1} & \leq(d-1) r^{-1}+1 \\
d(d-1) p^{-1} & \leq 2 q^{-1}+d(d-1) r^{-1}, \\
(d-2)(d+1) p^{-1} & \leq d(d-1) r^{-1} .
\end{aligned}
$$

The key exponents for this problem are

$$
p_{0}=q_{0}=\frac{d+2}{d}, \text { and } r_{0}=\frac{d^{2}+d-2}{d^{2}-d-2}=\frac{d^{2}-d}{d^{2}-d-2} \cdot q_{0} .
$$

The set of all triples $\left(p^{-1}, q^{-1}, r^{-1}\right)$ satisfying the above three necessary conditions equals the convex hull of the three points $(1,1,1),(0,0,0)$ and $\left(p_{0}{ }^{-1}, q_{0}{ }^{-1}, r_{0}{ }^{-1}\right)$,

Date: December 5, 2000.

The first author was supported in part by NSF grant DMS-9970660 and performed this research while on appointment as a Miller Research Professor in the Miller Institute for Basic Research in Science. 
together with all points $\left(p^{-1}, q^{-1}, r^{-1}\right)$ for which there exists a triple $\left(\tilde{p}^{-1}, \tilde{q}^{-1}, \tilde{r}^{-1}\right)$ in that convex hull satisfying $p \geq \tilde{p}, q \leq \tilde{q}$, and $r \leq \tilde{r}$.

Theorem 1. Let $d \geq 3$ be arbitrary, and let $I_{0}, C_{0}$ be fixed. Then $X$ maps $L^{p}\left(\mathbb{R}^{d}\right)$ to $L^{q}\left(L^{r}\right)(G)$ whenever $\left(p^{-1}, q^{-1}, r^{-1}\right)$ satisfies (3), (4), and (5) with strict inequality in each.

We will prove a sharper restricted weak type inequality:

$$
\left.\langle g, X f\rangle \leq C\|f\|_{L^{p}}\|g\|_{L^{q^{\prime}\left(L^{r^{\prime}}\right.}}\right)
$$

whenever $f, g$ are characteristic functions of measurable sets, for all $(p, q, r)$ satisfying (3), (4), and (5), where $q^{\prime}, r^{\prime}$ are the exponents conjugate respectively to $q, r$. By a crude interpolation argument, this implies boundedness from $L^{p}$ to $L^{q}\left(L^{r}\right)$ except at endpoints, as stated above; details are left to the reader.

The result is true, and is already well known, for $d=2$, where one is dealing with the full complex of all lines in $\mathbb{R}^{2}$, and the critical exponent $r_{0}$ equals $\infty$. Our arguments can be adapted to handle $d=2$, as well, although the details will not be given here.

This theorem was previously established by Wolff $^{1}[12]$ for $d=3$, and by Erdoğan [3] for $d=4,5$. The latter analysis suggested that the treatment of higher-dimensional cases would require higher-order versions of the "bush" and "hairbrush" constructions of Bourgain [1] and Wolff [10]. The approach of [2] may be viewed as a systematic version of such constructions, of arbitrary order; it also has a more algebraic perspective, quantified for the present situation in Lemma 4, which seems to be useful in high dimensions.

For purposes of exposition, we first discuss the simpler case of ordinary Lebesgue space esimates, $q=r$. Define $\Delta_{d}$ to be the set of all $\left(p^{-1}, q^{-1}\right) \in[0,1]^{2}$ that belong to the convex hull of the three points $(1,1),(0,0)$, and $\left(p_{1}^{-1}, q_{1}^{-1}\right)$, where $p_{1}=$ $d(d+1) /\left(d^{2}-d+2\right)$ and $q_{1}=(d+1) /(d-1)$. Examples discussed in [3] demonstrate that $X$ is unbounded unless $\left(p^{-1}, q^{-1}\right) \in \Delta_{d}$.

Theorem 2. $X$ maps $L^{p}\left(\mathbb{R}^{d}\right)$ to $L^{q}(G)$ for all $\left(p^{-1}, q^{-1}\right) \in \Delta_{d}$, except possibly for $\left(p_{1}{ }^{-1}, q_{1}^{-1}\right)$. It is of restricted weak type $\left(p_{1}, q_{1}\right)$.

Modulo the algebraic calculation in Lemma 4 below, this theorem is a direct consequence of the method developed in [2]. Various partial results can be found in $[4,5,6,7,9]$.

We begin with some preliminaries. It will be convenient to reparametrize $G$. Instead of taking $V_{\theta}$ to be the orthocomplement of $\nu_{\theta}$, we instead replace it by a fixed copy of $\mathbb{R}^{d-1}$, namely $V=\left\{y \in \mathbb{R}^{d}: y_{1}=0\right\}$. Thus $G$ will be identified with a compact subset of $\mathbb{R} \times \mathbb{R}^{d-1}=\mathbb{R} \times V$. Define for $(\theta, y) \in G$ and $s \in \mathbb{R}$

$$
\gamma(\theta, y, s)=\left(s, y_{2}+s \theta, \ldots, y_{d}+s \theta^{d-1}\right) .
$$

Then $X$ may be identified with the operator

$$
X f(\theta, y)=\int_{\mathbb{R}} f(\gamma(\theta, y, s)) d s
$$

\footnotetext{
${ }^{1}$ Wolff analyzed a related family of operators in all dimensions, which essentially coincides with $X$ in dimension three, but not otherwise; in higher dimensions he obtained partial results.
} 
where it is always understood that the integration with respect to $s$ is taken over a fixed compact subset of $\mathbb{R}$. Mixed norms are now

$$
\|g\|_{L^{q}\left(L^{r}\right)(G)}=\left(\int\left(\int_{V}|g(\theta, y)|^{r} d y\right)^{q / r} d \theta\right)^{1 / q} .
$$

The adjoint operator defined by $\int_{G} X f \cdot g=\int_{\mathbb{R}^{d}} f \cdot X^{*} g$ is

$$
X^{*} g(x)=\int_{\mathbb{R}} g\left(\gamma^{*}(x, t)\right) d t
$$

where

$$
\gamma^{*}(x, t)=\left(t, x_{2}-x_{1} t, x_{3}-x_{1} t^{2}, \ldots, x_{d}-x_{1} t^{d-1}\right) .
$$

We denote the characteristic function of a set $E$ by $\chi_{E}$. For any two measurable sets $E \subset \mathbb{R}^{d}, F \subset G$, define

$$
\mathcal{X}(E, F)=\left\langle\chi_{F}, X\left(\chi_{E}\right)\right\rangle
$$

where the inner product denotes here integration of $\chi_{F} \cdot X\left(\chi_{E}\right)$ over $G$. This bilinear form may be written in a formally symmetric way as $\left\langle X^{*}\left(\chi_{F}\right), \chi_{E}\right\rangle$; this symmetry manifests itself in the proof below. Fix any two such measurable sets having finite measures. Define two quantities $\alpha, \beta$ by

$$
\begin{aligned}
\alpha & =\mathcal{X}(E, F) /|F| \\
\beta & =\mathcal{X}(E, F) /|E| .
\end{aligned}
$$

We aim to show that

$$
\begin{aligned}
& |E| \geq c \alpha^{d} \beta^{d(d-1) / 2} \text { for } d \text { even, } \\
& |F| \geq c \alpha^{d-1} \beta^{\left(d^{2}-d+2\right) / 2} \text { for } d \text { odd. }
\end{aligned}
$$

for some fixed constant $c>0$. From this, Theorem 2 follows directly. Indeed, substituting for $\alpha, \beta$ for $d$ even gives

$$
|E| \geq c \mathcal{X}(E, F)^{d(d+1) / 2}|F|^{-d}|E|^{-d(d-1) / 2},
$$

or equivalently,

$$
\mathcal{X}(E, F) \leq C|E|^{\left(d^{2}-d+2\right) / d(d+1)}|F|^{2 /(d+1)} .
$$

This means precisely that $X$ is of restricted weak type $\left(p_{1}, q_{1}\right)$. The case of odd $d$ is similar.

Define a sequence of maps $\Phi_{k}$ mapping $\mathbb{R}^{k}$ to $\mathbb{R}^{d}$ if $k$ is even, and to $G$ if $k$ is odd, by fixing a point $x_{0} \in \mathbb{R}^{d}$, defining $\Phi_{1}\left(t_{1}\right)=\gamma^{*}\left(x_{0}, t_{1}\right), \Phi_{2}\left(t_{1}, s_{1}\right)=\gamma\left(\Phi_{1}\left(t_{1}\right), s_{1}\right)$, and in general

$$
\begin{aligned}
\Phi_{2 k+1}\left(t_{1}, s_{1}, t_{2}, s_{2}, \ldots, t_{k+1}\right) & =\gamma^{*}\left(\Phi_{2 k}\left(t_{1}, s_{1}, t_{2}, s_{2}, \ldots, t_{k}, s_{k}\right), t_{k+1}\right), \\
\Phi_{2 k+2}\left(t_{1}, s_{1}, t_{2}, s_{2}, \ldots, t_{k+1}, s_{k+1}\right) & =\gamma\left(\Phi_{2 k+1}\left(t_{1}, s_{1}, t_{2}, s_{2}, \ldots, t_{k+1}\right), s_{k+1}\right) .
\end{aligned}
$$

The starting point of the analysis is a small lemma from [2].

Lemma 3. There exists $c>0$ such that for any measurable sets $E, F$ having finite measures, there exist $x_{0} \in E$ and a sequence of sets $\Omega_{k} \subset \mathbb{R}^{k}$ defined for $1 \leq k \leq d$ and having the following properties: 
(1) For each $k, \Omega_{k+1} \subset \Omega_{k} \times \mathbb{R}$

(2) $\left|\Omega_{1}\right| \geq c \beta$

(3) For $k$ even, for each point $\omega \in \Omega_{k},\left|\left\{t \in \mathbb{R}:(\omega, t) \in \Omega_{k+1}\right\}\right| \geq c \beta$

(4) For $k$ odd, for each point $\omega \in \Omega_{k},\left|\left\{s \in \mathbb{R}:(\omega, s) \in \Omega_{k+1}\right\}\right| \geq c \alpha$

(5) $\Phi_{k}\left(\Omega_{k}\right) \subset E$ for even $k$, and $\Phi_{k}\left(\Omega_{k}\right) \subset F$ for odd $k$

We denote coordinates in $\Omega_{d}$ by $(s, t)=\left(t_{1}, s_{1}, \ldots, t_{D}, s_{D}\right)$ for $d=2 D$ and $(s, t)=$ $\left(t_{1}, s_{1}, \ldots, t_{D}, s_{D}, t_{D+1}\right)$ for $d=2 D+1$.

To bring particular properties of our line complex into play, we compute the iterated mapping $\Phi_{d}$ :

$$
\begin{aligned}
& \Phi_{d}\left(x, t_{1}, s_{1}, \ldots, t_{D}, s_{D}\right) \\
& =\left(s_{D}, x_{2}+\sum_{j=1}^{D}\left(s_{j}-s_{j-1}\right) t_{j}, x_{3}+\sum_{j=1}^{D}\left(s_{j}-s_{j-1}\right) t_{j}^{2}, \ldots, x_{d}+\sum_{j=1}^{D}\left(s_{j}-s_{j-1}\right) t_{j}^{d-1}\right) \\
& \Phi_{d}\left(x, t_{1}, s_{1}, \ldots, t_{D}, s_{D}, t_{D+1}\right) \\
& =\left(t_{D+1}, x_{2}+\sum_{j=1}^{D}\left(s_{j}-s_{j-1}\right) t_{j}-s_{D} t_{D+1}, \ldots, x_{d}+\sum_{j=1}^{D}\left(s_{j}-s_{j-1}\right) t_{j}^{d-1}-s_{D} t_{D+1}^{d-1}\right)
\end{aligned}
$$

Here we have written $s_{0}=x_{1}$, the first coordinate of $x$, in order to simplify slightly the expression. Its Jacobian matrix $J(s, t)=\partial \Phi_{d} / \partial(s, t)$ of first partial derivatives with respect to $(s, t)$ equals

$$
\left(\begin{array}{ccccc}
1 & t_{D} & t_{D}^{2} & \cdots & t_{D}^{d-1} \\
0 & s_{D}-s_{D-1} & \left(s_{D}-s_{D-1}\right) 2 t_{D} & \cdots & \left(s_{D}-s_{D-1}\right)(d-1) t_{D}^{d-2} \\
0 & t_{D-1}-t_{D} & t_{D-1}^{2}-t_{D}^{2} & \cdots & t_{D-1}^{d-1}-t_{D}^{d-1} \\
0 & s_{D-1}-s_{D-2} & \left(s_{D-1}-s_{D-2}\right) 2 t_{D-1} & \cdots & \left(s_{D-1}-s_{D-2}\right)(d-1) t_{D-1}^{d-2} \\
\vdots & \vdots & \vdots & \vdots & \vdots \\
0 & t_{1}-t_{2} & t_{1}^{2}-t_{2}^{2} & \cdots & t_{1}^{d-1}-t_{2}^{d-1} \\
0 & s_{1}-s_{0} & \left(s_{1}-s_{0}\right) 2 t_{1} & \cdots & \left(s_{1}-s_{0}\right)(d-1) t_{1}^{d-2}
\end{array}\right)
$$

for $d=2 D$, and it equals

$$
\left(\begin{array}{ccccc}
1 & -s_{D} & -2 s_{D} t_{D+1} & \cdots & -(d-1) s_{D} t_{D+1}^{d-2} \\
0 & t_{D}-t_{D+1} & t_{D}^{2}-t_{D+1}^{2} & \cdots & t_{D}^{d-1}-t_{D+1}^{d-1} \\
0 & s_{D}-s_{D-1} & \left(s_{D}-s_{D-1}\right) 2 t_{D} & \cdots & \left(s_{D}-s_{D-1}\right)(d-1) t_{D}^{d-2} \\
0 & t_{D-1}-t_{D} & t_{D-1}^{2}-t_{D}^{2} & \cdots & t_{D-1}^{d-1}-t_{D}^{d-1} \\
0 & s_{D-1}-s_{D-2} & \left(s_{D-1}-s_{D-2}\right) 2 t_{D-1} & \cdots & \left(s_{D-1}-s_{D-2}\right)(d-1) t_{D-1}^{d-2} \\
\vdots & \vdots & \vdots & \vdots & \vdots \\
0 & t_{1}-t_{2} & t_{1}^{2}-t_{2}^{2} & \cdots & t_{1}^{d-1}-t_{2}^{d-1} \\
0 & s_{1}-s_{0} & \left(s_{1}-s_{0}\right) 2 t_{1} & \cdots & \left(s_{1}-s_{0}\right)(d-1) t_{1}^{d-2}
\end{array}\right)
$$

for $d=2 D+1$. 


\section{Lemma 4.}

$$
\begin{aligned}
& J=c_{d} \prod_{j=1}^{D}\left(s_{j}-s_{j-1}\right) \prod_{1 \leq j<k \leq D}\left(t_{j}-t_{k}\right)^{4} \quad \text { for } d=2 D \\
& J=c_{d} \prod_{j=1}^{D}\left(s_{j}-s_{j-1}\right) \prod_{1 \leq j<k \leq D}\left(t_{j}-t_{k}\right)^{4} \prod_{j=1}^{D}\left(t_{j}-t_{D+1}\right)^{2} \quad \text { for } d=2 D+1
\end{aligned}
$$

for some nonzero constants $c_{d}$.

Proof of Theorem 2. (i) Case 1: $d=2 D$. We have $E \supset \Phi_{d}\left(\Omega_{d}\right)$, and moreover, as shown in [2], it is a consequence of Bezout's theorem that

$$
\left|\Phi_{d}\left(\Omega_{d}\right)\right| \geq \frac{1}{d !} \int_{\Omega_{d}}|J(t, s)| d t d s
$$

where $(t, s)=\left(t_{1}, s_{1}, \ldots, t_{D}, s_{D}\right)$.

From the structural properties of the sets $\Omega_{k}$, it follows easily as in [2] and the proof below of (44) that

$$
\int_{\Omega_{d}}|J(s, t)| d s d t=c_{d} \int_{\Omega_{d}} \prod_{j=1}^{D}\left|s_{j}-s_{j-1}\right| \cdot \prod_{1 \leq j<k \leq D}\left|t_{j}-t_{k}\right|^{4} d s d t \geq c \alpha^{d} \beta^{d(d-1) / 2}
$$

This is (15), from which Theorem 2 follows directly, as shown above.

(ii) Case 2: $d=2 D+1$. The proof is essentially the same. We have $F \supset \Phi_{d}\left(\Omega_{d}\right)$, and as above

$$
\left|\Phi_{d}\left(\Omega_{d}\right)\right| \geq \frac{1}{d !} \int_{\Omega_{d}}|J(t, s)| d t d s
$$

where $(t, s)=\left(t_{1}, s_{1}, \ldots, t_{D}, s_{D}, t_{D+1}\right)$. Proceeding as above, we obtain

$$
\begin{aligned}
\int_{\Omega_{d}}|J(s, t)| d s d t & =c_{d} \int_{\Omega_{d}} \prod_{j=1}^{D}\left|s_{j}-s_{j-1}\right| \cdot \prod_{1 \leq j<k \leq D}\left|t_{j}-t_{k}\right|^{4} \prod_{j=1}^{D}\left(t_{j}-t_{D+1}\right)^{2} d s d t \\
& \geq c \alpha^{d-1} \beta^{\left(d^{2}-d+2\right) / 2}
\end{aligned}
$$

which completes the proof of the Theorem.

Proof of Lemma 4. (i) Case 1: $d=2 D . J$ is a polynomial of total degree $d(d-1) / 2$. The right-hand side of (21) has the same degree:

$$
\frac{d}{2}+4 \frac{1}{2} \frac{d}{2}\left(\frac{d}{2}-1\right)=\frac{d+d(d-2)}{2}=\frac{d(d-1)}{2} .
$$

Clearly $J$ is divisible by $s_{j}-s_{j-1}$ for all $j \geq 1$, so it suffices merely to show that it is divisible by $\left(t_{j}-t_{k}\right)^{4}$ for arbitrary $j \neq k$.

To do so, factor $\left(s_{j}-s_{j-1}\right)$ out from each row where it occurs, leaving a matrix involving only $t_{1}, \ldots, t_{D}$. Fix any $j \neq k$. Perform row operations to transform this 
matrix to one whose first three rows are

$$
\left(\begin{array}{ccccc}
t_{k}^{0} & 2 t_{k} & 3 t_{k}^{2} & \cdots & (d-1) t_{k}^{d-2} \\
t_{j}-t_{k} & t_{j}^{2}-t_{k}^{2} & t_{j}^{3}-t_{k}^{3} & \cdots & t_{j}^{d-1}-t_{k}^{d-1} \\
t_{j}^{0} & 2 t_{j} & 3 t_{j}^{2} & \cdots & (d-1) t_{j}^{d-2}
\end{array}\right) .
$$

It therefore suffices to show that the determinant of any three by three submatrix

$$
\left(\begin{array}{ccc}
\mu t_{k}^{\mu-1} & \nu t_{k}^{\nu-1} & \rho t_{k}^{\rho-1} \\
t_{j}^{\mu}-t_{k}^{\mu} & t_{j}^{\nu}-t_{k}^{\nu} & t_{j}^{\rho}-t_{k}^{\rho} \\
\mu t_{j}^{\mu-1} & \nu t_{j}^{\nu-1} & \rho t_{j}^{\rho-1}
\end{array}\right)
$$

is divisible by $\left(t_{j}-t_{k}\right)^{4}$. By homogeneity we may assume that $t_{j}=1$, and we then write $t_{k}=1+z$ and seek to show that this subdeterminant is $O\left(z^{4}\right)$ as $z \rightarrow 0$.

Multiplying the middle row by -1 , then adding the third row to the first, transforms the preceding matrix to

$$
\left(\begin{array}{ccc}
\mu(1+z)^{\mu-1}-\mu & \nu(1+z)^{\nu-1}-\nu & \rho(1+z)^{\rho-1}-\rho \\
(1+z)^{\mu}-1 & (1+z)^{\nu}-1 & (1+z)^{\rho}-1 \\
\mu & \nu & \rho
\end{array}\right)
$$

We expand in Taylor series about $z=0$. Since the first two rows vanish when $z=0$, all terms of degree $>2$ may be discarded; their contributions to the determinant will be $O\left(z^{4}\right)$. We also factor $\mu, \nu, \rho$ respectively out from the three columns to obtain

$$
\left(\begin{array}{ccc}
(\mu-1) z+O\left(z^{2}\right) & (\nu-1) z+O\left(z^{2}\right) & (\rho-1) z+O\left(z^{2}\right) \\
z+\frac{1}{2}(\mu-1) z^{2} & z+\frac{1}{2}(\nu-1) z^{2} & z+\frac{1}{2}(\rho-1) z^{2} \\
1 & 1 & 1
\end{array}\right)
$$

Subtracting $z$ times the third row from the second gives

$$
\left(\begin{array}{ccc}
(\mu-1) z+O\left(z^{2}\right) & (\nu-1) z+O\left(z^{2}\right) & (\rho-1) z+O\left(z^{2}\right) \\
\frac{1}{2}(\mu-1) z^{2} & \frac{1}{2}(\nu-1) z^{2} & \frac{1}{2}(\rho-1) z^{2} \\
1 & 1 & 1
\end{array}\right)
$$

In computing the coefficient of $z^{3}$ in the resulting determinant, we may drop the quadratic terms in the first row; the first two rows of the resulting matrix are clearly linearly dependent, so its determinant vanishes. Hence $J$ takes the form (21), for some scalar $c_{d}$.

To see that $c_{d} \neq 0$, we directly expand the determinant and find that the coefficient of $t_{d / 2}^{2 d-4} t_{(d / 2)-1}^{2 d-8} \cdots t_{2}^{4} t_{1}^{0}$ is a nonzero multiple of $\prod_{j}\left(s_{j}-s_{j-1}\right)$; hence $J$ does not vanish identically.

(ii) Case 2: $d=2 D+1$. As above, it suffices to show that $J$ is divisible by $\left(t_{j}-t_{k}\right)^{4}$ for $1 \leq j<k \leq D$ and by $\left(t_{j}-t_{D+1}\right)^{2}$ for $1 \leq j \leq D$. The proof of the former is essentially the same as above. We will prove that $J$ is divisible by $\left(t_{j}-t_{D+1}\right)^{2}$ for $1 \leq j \leq D$

To do so, factor $\left(s_{j}-s_{j-1}\right)$ out from each row where it occurs, leaving a matrix involving only $t_{1}, \ldots, t_{D+1}$. Fix any $j \leq D$. Perform row operations to transform this 
matrix to one whose first two rows are

$$
\left(\begin{array}{ccccc}
t_{j}-t_{D+1} & t_{j}^{2}-t_{D+1}^{2} & t_{j}^{3}-t_{D+1}^{3} & \cdots & t_{j}^{d-1}-t_{D+1}^{d-1} \\
t_{j}^{0} & 2 t_{j} & 3 t_{j}^{2} & \cdots & (d-1) t_{j}^{d-2}
\end{array}\right) .
$$

As above, it suffices to show that the determinant of any two by two submatrix of $(32)$ is divisible by $\left(t_{j}-t_{D+1}\right)^{2}$. This can easily be seen by factoring out $\left(t_{j}-t_{D+1}\right)$ from the first row and observing that the determinant of the remaining matrix vanishes then $t_{D}=t_{D+1}$.

The proof of Theorem 1 will require the conversion of information expressed in terms of $|F|$ to mixed norms of $\chi_{F}$. The next lemma suffices for that purpose. For any set $F \subset G=\mathbb{R}^{1} \times \mathbb{R}^{d-1}$, denote by $\pi(F)$ its projection onto the first factor $\mathbb{R}^{1}$.

\section{Lemma 5.}

$$
|F| \leq\left\|\chi_{F}\right\|_{L^{q_{0}^{\prime}}\left(L^{r_{0}^{\prime}}\right)}^{(d+2)(d-1) / 2 d}|\pi(F)|^{1 / d} .
$$

Proof. Writing $f(\theta)=\left|\left\{y \in \mathbb{R}^{d-1}:(\theta, y) \in F\right\}\right|$, we have

$$
\left\|\chi_{F}\right\|_{L^{q_{0}^{\prime}\left(L^{r_{0}^{\prime}}\right)}}=\left(\int_{\pi(F)} f(\theta)^{q_{0}^{\prime} / r_{0}^{\prime}} d \theta\right)^{1 / q_{0}^{\prime}}
$$

and $|F|=\int_{\pi(F)} f$. By Hölder,

$$
|F| \leq|\pi(F)|^{1 / d}\left(\int_{\pi(F)} f^{d /(d-1)}\right)^{(d-1) / d} .
$$

Now $\frac{q_{0}^{\prime}}{r_{0}^{\prime}}=\frac{d+2}{2} \cdot \frac{2 d}{d^{2}+d-2}=\frac{d}{d-1}$, so the right-hand side of the last inequality is precisely the right-hand side of (33).

To prove Theorem 1, it suffices to establish the restricted weak type bound at the endpoint $\left(p_{0}{ }^{-1}, q_{0}{ }^{-1}, r_{0}{ }^{-1}\right): \mathcal{X}(E, F) \leq C|E|^{1 / p_{0}}\left\|\chi_{F}\right\|_{L^{q_{0}^{\prime}\left(L^{r_{0}^{\prime}}\right)}}$ for any measurable sets $E \subset \mathbb{R}^{d}, F \subset G$ having finite measure. Moreover, it suffices to prove this for any $F$ and any $\beta>0$, with

$$
E=\left\{x \in \mathbb{R}^{d}: \beta \leq X^{*}\left(\chi_{F}\right)(x)<2 \beta\right\} .
$$

One of the devices we will use is a stopping-time procedure inspired partly by an argument of Tao and Wright [8]. Let $\varepsilon>0$ be a small constant to be chosen later. It is possible to select for each $x$ a nonegative integer $m \leq C \log \left(\beta^{-1}\right)$, and an interval $I_{x} \subset \mathbb{R}$ of length $2^{m} \beta$, such that for any subinterval $I^{\prime} \subset I_{x}$ of length $2^{m-1} \beta$,

$$
\left|\left\{t \in I_{x} \backslash I^{\prime}: \gamma^{*}(x, t) \in F\right\}\right| \geq c 2^{-\varepsilon m} \beta,
$$

where $c>0$ is a fixed constant, depending only on the choice of $\varepsilon$.

Indeed, let $K_{0}$ be a fixed interval of length $2^{M} \beta \sim 1$, so that $\mid\left\{t \in K_{0}: \gamma^{*}(x, t) \in\right.$ $F\} \mid \geq \beta$. Consider any subintervals $K \subset K_{0}$ of length $2^{M-1} \beta$ satisfying $\mid\{t \in K$ : $\left.\gamma^{*}(x, t) \in F\right\} \mid \geq\left(1-2^{-\varepsilon M}\right) \beta$. If no such subinterval exists, then we set $I_{x}=K_{0}$. Otherwise we choose one such subinterval, call it $K_{1}$, and ask whether there exists an interval $K \subset K_{1}$ of length $2^{M-2} \beta$ for which $\left|\left\{t \in K: \gamma^{*}(x, t) \in F\right\}\right| \geq(1-$ 
$\left.2^{-\varepsilon(M-1)}\right)\left|\left\{t \in K_{1}: \gamma^{*}(x, t) \in F\right\}\right|$. If not, we set $I_{x}=K_{1}$. Continuing this way, we ask at step $m$ whether there exists $K \subset K_{m}$ of length $2^{m-1} \beta$ satisfying

$$
\left|\left\{t \in K: \gamma^{*}(x, t) \in F\right\}\right| \geq\left(1-2^{-\varepsilon m}\right)\left|\left\{t \in K_{m}: \gamma^{*}(x, t) \in F\right\}\right| .
$$

If not, we set $I_{x}=K_{m}$. If the selection process stops at step $m$ then we have $\left|I_{x}\right|=2^{m} \beta$ and for any subinterval $K \subset I_{x}$ of length $2^{m-1} \beta,\left|\left\{t \in I_{x}: \gamma^{*}(x, t) \in F\right\}\right| \geq$ $\left(1-2^{-\varepsilon M}\right)\left(1-2^{-\varepsilon(M-1)}\right) \cdots\left(1-2^{-\varepsilon(m+1)}\right) \beta \geq c \beta$ by induction, and consequently (37) holds by virtue of the selection rule (38). Since $c=c(\varepsilon) \rightarrow 1$ as $\varepsilon \rightarrow 0$, by choosing $\varepsilon$ to be sufficiently small we may ensure that the process does indeed stop with $\left|I_{x}\right| \geq \beta$, since otherwise we would obtain a subinterval $I^{\prime}$ of length $\beta / 2$ such that $\left|\left\{t \in I^{\prime}: \gamma^{*}(x, t) \in F\right\}\right| \geq(3 / 4) \beta>\left|I^{\prime}\right|$.

Set

$$
E^{m}=\left\{x \in E:\left|I_{x}\right|=2^{m} \beta\right\},
$$

for $0 \leq m \leq C \log (1 / \beta)$. Partition a compact subset of $\mathbb{R}$ into $\sim\left(2^{m} \beta\right)^{-1}$ intervals $J_{j}^{m}$ of lengths $2^{m} \beta$, and set

$$
\begin{aligned}
& E_{j}^{m}=\left\{x \in E^{m}: I_{x} \cap J_{j}^{m} \neq \emptyset\right\} \\
& F_{j}^{m}=F \cap \pi^{-1}\left(J_{j-1}^{m} \cup J_{j}^{m} \cup J_{j+1}^{m}\right) .
\end{aligned}
$$

The collections $\left\{E_{j}^{m}\right\}_{m, j}$ and $\left\{\pi\left(F_{j}^{m}\right)\right\}_{j}$ have bounded overlaps, in the sense that there exists an absolute constant $C<\infty$ such that no point of $E$ belongs to more than $C$ sets $E_{j}^{m}$, and for fixed $m$, no point of $\pi(F)$ belongs to more than $C$ sets $F_{j}^{m}$. Since $E^{m}=\cup_{j} E_{j}^{m},(36)$ and (37) imply that

$$
\mathcal{X}\left(E^{m}, F\right) \leq \sum_{j} \mathcal{X}\left(E_{j}^{m}, F\right) \leq 2 \beta \sum_{j}\left|E_{j}^{m}\right| \leq C 2^{\varepsilon m} \sum_{j} \mathcal{X}\left(E_{j}^{m}, F_{j}^{m}\right) .
$$

For each pair $F_{j}^{m}, E_{j}^{m}$ we associate to $\mathcal{X}\left(E_{j}^{m}, F_{j}^{m}\right)$ the two quantities

$$
\alpha_{j}^{m}=\mathcal{X}\left(E_{j}^{m}, F_{j}^{m}\right) /\left|F_{j}^{m}\right| \quad \text { and } \quad \beta_{j}^{m}=\mathcal{X}\left(E_{j}^{m}, F_{j}^{m}\right) /\left|E_{j}^{m}\right| .
$$

Now (37) guarantees that $\beta_{j}^{m} \geq c 2^{-\varepsilon m} \beta$, and $\beta_{j}^{m} \leq 2 \beta$ by the definition of $E$.

Fix $m, j$. By the proof of Lemma 3 , there exist sets $\Omega_{k}^{m, j}$, for $1 \leq k \leq d$, satisfying the conclusions of that lemma with $\alpha_{j}^{m}, \beta_{j}^{m}$ respectively replacing $\alpha, \beta$, and with the additional property that for all even $k$, for each $\omega \in \Omega_{k}^{m, j}$, for any subinterval $I$ of $\mathbb{R}$ having length $2^{m-1} \beta$,

$$
\left|\left\{t \notin I:(\omega, t) \in \Omega_{k+1}^{m, j}\right\}\right| \geq c 2^{-\varepsilon m} \beta .
$$

\section{Lemma 6.}

$$
\begin{aligned}
& \int_{\Omega_{d}^{m, j}}|J| d s d t \geq c 2^{2 m}\left(\alpha_{j}^{m}\right)^{d} \cdot\left(\beta_{j}^{m}\right)^{d(d-1) / 2} \text { for d even, } \\
& \int_{\Omega_{d}^{m, j}}|J| d s d t \geq c 2^{2 m}\left(\alpha_{j}^{m}\right)^{d-1} \cdot\left(\beta_{j}^{m}\right)^{\left(d^{2}-d+2\right) / 2} \text { for } d \text { odd } .
\end{aligned}
$$


Proof. We will give the proof only for the case of even $d$, and will first indicate how to obtain the estimate without the bonus factor of $2^{m}$. To simplify notation we drop the superscripts and write simply $\Omega_{k}=\Omega_{k}^{m, j}$. With $D=d / 2, \int_{\Omega_{d}}|J|$ equals

$$
c_{d} \int_{\Omega_{d-1}} \prod_{j<D}\left|s_{j}-s_{j-1}\right| \cdot \prod_{j<k}\left|t_{j}-t_{k}\right|^{4} \int_{\mathbb{R}}\left|s_{D}-s_{D-1}\right| d s_{D} d \omega
$$

where $\omega=\left(t_{1}, s_{1}, \ldots, s_{D-1}, t_{D}\right) \in \Omega_{d-1}$. In the inner integral, $s_{D}$ varies over a set of measure at least $c \alpha_{j}^{m}$, while $s_{D-1}$ remains fixed. Therefore the factor $\left|s_{D}-s_{D-1}\right|$ is $\geq c^{\prime} \alpha_{j}^{m}$ on a subset of measure at least $c^{\prime \prime} \alpha_{j}^{m}$. Thus the inner integral is bounded below by a constant times $\left(\alpha_{j}^{m}\right)^{2}$.

We repeat the procedure with respect to the next coordinate, $t_{D}$, finding that $(45)$ is

$$
\geq c\left(\alpha_{j}^{m}\right)^{2} \int_{\Omega_{d-2}} \prod_{j<D}\left|s_{j}-s_{j-1}\right| \cdot \prod_{j<k<D}\left|t_{j}-t_{k}\right|^{4} \int_{\mathbb{R}} \prod_{j<D}\left|t_{D}-t_{j}\right|^{4} d t_{D} d \omega
$$

where now $\omega=\left(t_{1}, s_{1}, \ldots, t_{D-1}, s_{D-1}\right)$. For fixed $\omega, t_{D}$ varies over a set whose measure is $\geq c \beta_{j}^{m}$. Moreover, on at least a fixed fraction of that set, each factor $\left|t_{D}-t_{j}\right|$ is $\geq c \tilde{\beta}$. It results that $(46)$ is

$$
\geq c\left(\alpha_{j}^{m}\right)^{2} \beta_{j}^{m} \cdot(\beta)^{2 d-4} \int_{\Omega_{d-2}} \prod_{j<D}\left|s_{j}-s_{j-1}\right| \cdot \prod_{j<k<D}\left|t_{j}-t_{k}\right|^{4} .
$$

Iterating this reasoning leads to (44), except for the factor of $2^{2 m}$.

To obtain it, reconsider the step involving an integration with respect to $t_{2}$. For any $\left(t_{1}, s_{1}\right) \in \Omega_{2}$, because of (43), the factor $\left|t_{2}-t_{1}\right|$ in the Jacobian is bounded below by $c 2^{m} \beta$ for a set of values of $t_{2}$ having measure $\geq 2^{-\varepsilon m} \beta_{j}^{m}$. This factor is raised to either the power 2 or 4 , resulting in an improvement by a factor $\geq 2^{2 m}$.

(44) is fundamental because $\int_{\Omega_{d}^{m, j}}|J| d s d t \leq C\left|E_{j}^{m}\right|$ for $d$ even, and is $\leq C\left|F_{j}^{m}\right|$ for $d$ odd. Thus, for even $d$,

$$
\begin{aligned}
& \left|E_{j}^{m}\right| \geq c 2^{2 m}\left(\alpha_{j}^{m}\right)^{d}\left(\beta_{j}^{m}\right)^{d(d-1) / 2} \text { for } d \text { even } \\
& \left|F_{j}^{m}\right| \geq c 2^{2 m}\left(\alpha_{j}^{m}\right)^{d-1}\left(\beta_{j}^{m}\right)^{\left(d^{2}-d+2\right) / 2} \text { for } d \text { odd. }
\end{aligned}
$$

Since $\beta_{j}^{m} \geq 2^{-\varepsilon m} \beta$ and since we may choose $\varepsilon \leq 1 / 2$, we may rewrite these as

$$
\begin{aligned}
& \left|E_{j}^{m}\right| \geq c 2^{m / 2}\left(\alpha_{j}^{m}\right)^{d}\left(\beta_{j}^{m}\right)^{\left(d^{2}-d-2\right) / 2}\left(2^{m} \beta\right) \text { for } d \text { even } \\
& \left|F_{j}^{m}\right| \geq c 2^{m / 2}\left(\alpha_{j}^{m}\right)^{d-1}\left(\beta_{j}^{m}\right)^{\left(d^{2}-d\right) / 2}\left(2^{m} \beta\right) \text { for } d \text { odd. }
\end{aligned}
$$

Substituting for $\alpha_{j}^{m}, \beta_{j}^{m}$ according to their definitions, we obtain after a short calculation that

$$
\mathcal{X}\left(E_{j}^{m}, F_{j}^{m}\right) \leq C 2^{-\delta m}\left|E_{j}^{m}\right|^{d /(d+2)}\left|F_{j}^{m}\right|^{2 d /(d+2)(d-1)} \cdot\left(2^{m} \beta\right)^{-2 /(d+2)(d-1)}
$$

for some positive constants $C<\infty, \delta>0$.

To express this as a mixed norm estimate, recall that the projection $\pi\left(F_{j}^{m}\right)$ of $F_{j}^{m} \subset \mathbb{R}^{1} \times \mathbb{R}^{d-1}$ onto the first factor, $\mathbb{R}^{1}$, satisfies $\left|\pi\left(F_{j}^{m}\right)\right| \leq 3 \cdot 2^{m} \beta$, simply because 
$\pi\left(F_{j}^{m}\right) \subset J_{j-1}^{m} \cup J_{j}^{m} \cup J_{j+1}^{m}$ and each of the latter intervals has length $2^{m} \beta$. Returning to $(50)$, we thus obtain

$$
\begin{aligned}
\mathcal{X}\left(E_{j}^{m}, F_{j}^{m}\right) & \leq C 2^{-\delta m}\left|E_{j}^{m}\right|^{d /(d+2)}\left(\left|F_{j}^{m}\right| \cdot\left|\pi\left(F_{j}^{m}\right)\right|^{-1 / d}\right)^{2 d /(d+2)(d-1)} \\
& \leq C 2^{-\delta m}\left|E_{j}^{m}\right|^{d /(d+2)}\left\|\chi_{F_{j}^{m}}\right\|_{L^{q^{\prime}\left(L^{r^{\prime}}\right)}}
\end{aligned}
$$

to obtain the last line we have applied (33), with $q=q_{0}, r=r_{0}$.

Writing $p=p_{0}=q_{0}=(d+2) / d$ and invoking Hölder's inequality yields

$$
\begin{aligned}
\sum_{j} \mathcal{X}\left(E_{j}^{m}, F_{j}^{m}\right) & \leq C 2^{-\delta m}\left(\sum_{j}\left|E_{j}^{m}\right|\right)^{1 / p}\left(\sum_{j}\left\|\chi_{F_{j}^{m}}\right\|_{L^{q^{\prime}\left(L^{r^{\prime}}\right)}}^{q^{\prime}}\right)^{1 / q^{\prime}} \\
& \leq C 2^{-\delta m}|E|^{1 / p}\left\|\chi_{F}\right\|_{L^{q^{\prime}}\left(L^{r^{\prime}}\right)} ;
\end{aligned}
$$

to obtain the final inequality we have used the bounded overlap property of the collection $\left\{\pi\left(F_{j}^{m}\right): j=1,2, \ldots\right\}$ of projections, uniformly in $m$. Since $E=\cup_{m} E^{m}$, and since $\delta$ is independent of $\varepsilon$, by choosing $\varepsilon<\delta$ we may ensure that the factor of $2^{-\delta m}$ here more than compensates for the loss of $2^{\varepsilon m}$ in (41). Therefore summation over $m \geq 0$ gives

$$
\mathcal{X}(E, F) \leq C|E|^{1 / p}\left\|\chi_{F}\right\|_{L^{q^{\prime}}\left(L^{r^{\prime}}\right)},
$$

concluding the proof of Theorem 1.

A final remark: The argument, as given, does not apply in the two-dimensional case, where one is dealing with the Kakeya maximal function and the exponent $r_{0}$ equals $\infty$. The difficulty is that in order to gain the factor of $2^{2 m}$ in (44), we needed to have at least two variables $t_{j}$. Hence $\left|\pi\left(F_{j}^{m}\right)\right|$ cannot be brought into play in the same way when $d=2$. Nonetheless, the argument can be applied after slight reorganization.

\section{REFERENCES}

[1] J. Bourgain, Besicovitch type maximal operators and applications to Fourier analysis, Geometric and Functional Analysis 1 (1991), 147-187.

[2] M. Christ, Convolution, curvature, and combinatorics. A case study, Internat. Math. Research Notices 19 (1998), 1033-1048.

[3] M. B. Erdoğan, Mixed norm estimates for a restricted X-ray transform in $\mathbb{R}^{4}$ and $\mathbb{R}^{5}$, preprint.

[4] A. Greenleaf and A. Seeger, Fourier integral operators with fold singularities, J. Reine Angew. Math. 455 (1994), 35-56.

[5] _ Fourier integral operators with cusp singularities, Amer. Jour. Math. 120 (1998), 10771119.

[6] On X-ray transforms for rigid line complexes and integrals over curves in $\mathbb{R}^{4}$, Proc. Amer. Math. Soc. 127 (1999), 3533-3545.

[7] D. Oberlin, An estimate for a restricted $X$-ray transform, preprint.

[8] T. Tao, personal communication.

[9] H. T. Wang, $L^{p}$ estimates for the X-ray transform restricted to line complexes of Kirillov type, Trans. Amer. Math. Soc. 332 (1992), 793-821.

[10] T. Wolff, An improved bound for Kakeya type maximal functions, Revista Mat. Iberoamericana 11 (1995), 651-674.

[11] _ A mixed norm estimate for the X-ray transform, Revista Mat. Iberoamericana 14 (1998), 561-601. 
[12] _ A sharp bilinear cone restriction estimate, preprint.

Michael Christ, Department of Mathematics, University of California, Berkeley, CA 94720-3840, USA

E-mail address: mchrist@math.berkeley.edu

M. Burak Erdoğan, Department of Mathematics, California Institute of TechNOLOGy, PASADENa, CA 91125, USA

E-mail address: mehmet@its.caltech.edu 